\title{
Image Compression with Contourlet Transformer and Quantitative Evaluation by using WVT
}

\author{
Miss. Sneha Shukla ${ }^{1}$, Prof. Nilesh Bodne ${ }^{2}$, Prof. Pranjali Dahikar ${ }^{3}$ \\ Electronics and Communication, VIT, Umred Road, Nagpur, India ${ }^{1,2,3}$
}

\begin{abstract}
In this paper wavelet based method have expanded in the field of still image, they offer the advantage of a better trade of between complexity, Compression and quality over the tradition DCT - based method, However for image compression WVT has a problem with the orientation selectively. It does not represent two dimensional Singularities effectively, to overcome this problem used Contourlet transform for image compression. It can better represent curve and edge of two dimensional images. CTT is a multi-scale and directional de-composition of a signal using a combination of modified LP and a directional filter bank. CTT can be applied efficiently to capture smooth contours at larger resolutions, while WVT can be used for lower resolution images for further information compaction. A comparative study is performed between the contourlet and the Wavelet analysis in terms of result quality and information compaction using a new metric.
\end{abstract}

Keywords: Transform, Contourlet Transform, De-composition, Directional Filter Bank

\section{INTRODUCTION}

During the past two decades, image compression has developed from a mostly academic Rate Distortion field, into a highly commercial business. Various lossless and lossy image coding techniques have been developed. Since the compression ratio obtainable from lossy compression can significantly exceed that obtainable from lossless compression, the primary trade-off concerns the need for reproducibility versus the storage and transmission requirements. Lossy compression mainly consists of de-correlation and quantization stages that reduce the image size by permanently eliminating certain information. One approach is the use of multi resolution transforms, which are free from blocking effect artefacts such as in case of the Discrete Cosine Transform (DCT), which is used in the JPEG (baseline) industry standard. By the use of the WaVelet Transform (WVT), the corresponding coefficients of the different decomposition levels are correlated and show a characteristic trend. This residual correlation is indicative for a further compression potential. Some standard methods get profits from this potential, especially when considering sets of transform coefficients as feature specific compounds. Wavelet-based methods have expanded in the field of still image and video compression; they offer the advantage of a better trade-off between complexity, compression and quality over the traditional DCT-based methods. However, for image compression, WVT has a problem with the orientation selectivity because it provides local frequency representation of image regions over a range of spatial scales, and therefore, it does not represent two-dimensional singularities effectively. In a map of the large wavelet coefficient, one sees the edges of the images repeated at scale after scale. In this paper, we used Contourlet transform To avoid the local frequency representation in wavelet transformer.

\section{RELATED WORK}

\section{A. Wavelet Transforms}

Wavelets have been recognized as the correct tool for representing the one-dimensional piecewise smooth signals, because wavelets provide an optimum illustration for these signals in a certain sense Also, the wavelet representation is responsive to efficient algorithms; in particular it leads to fast transforms and convenient tree data structures.. Wavelet analysis is very powerful and extremely useful for compressing data such as image and lot of work has been done in the area of wavelet based lossy image compression. It's power comes from its multi-resolution. "Wavelet partitions plane into congruent four-sided figure of square sided length i.e. variable length and variable width that are related by and constructs a system of renormalized Wavelets smoothly localized near each four-sided figure" as a consequence Wavelet transform involves square scaling. In this paper Dual Tree Complex Wavelet Transforms (DTCWT) is considered that entails two filters namely analysis filter bank and synthesis filter bank. By Analysis filter bank, signal is decomposed into low pass filtered coefficients and high pass filtered coefficients and each of these filtered are down sampled by 2 , hence wavelet coefficients are obtained. By synthesis filter bank, these wavelet coefficients are up sampled by 2 and reconstructed into a signal. Wavelet function, forward Wavelet transform and Inverse Wavelet transform are defined by expressions, (1), (2) and (3) respectively. 


$$
\begin{gathered}
\psi_{a, b}(x)=\frac{1}{\sqrt{a}} \psi\left(\frac{x-b}{a}\right) \\
W_{T}(a, b)=\int f(x) \frac{1}{\sqrt{a}} \psi\left(\frac{x-b}{a}\right) d t \\
f(x)=\int_{-\infty}^{\infty} \int_{0}^{\infty} W_{T}(a, b) \psi_{a, b}(x) d a d b
\end{gathered}
$$

Here, $a$ is Scaling parameter and $b$ is translation parameter.

\section{B. Contourlet Transforms}

The Contourlet Transform presented in [8], facilitates to perform critical sampling at different directions and at each level or resolution. It can represent the images with less redundancy and significant approximation properties for smooth 2D functions, and is computationally competent enough to capture the contours and fine details in the image for image compression applications. Efficient representations of signals require that coefficients of functions, which represent the regions of interest, are sparse. Wavelets can pick up discontinuities of one dimensional piecewise smooth functions very efficiently and rep-resent them as point discontinuities. 2D WVT obtained by a tensor product of onedimensional wavelets are good to isolate discontinuities at edge points, but cannot recognize smoothness along contours. Numerous methods were developed to overcome this by adaptive, Radon-based, or filter bank-based techniques. Do and Vetterli pro-posed the Pyramidal Directional Filter-Bank (PDFB), which overcomes the blockbased approach of CVT by a directional filter bank, applied on the whole scale, also known as CTT. It has been developed to offer the directionality and anisotropy to image representation that are not provided by separable WVT. CTT is a multi-scale and directional de-composition of a signal using a combination of a modified Laplacian Pyramid (LP) and a Directional Filter Bank (DFB).
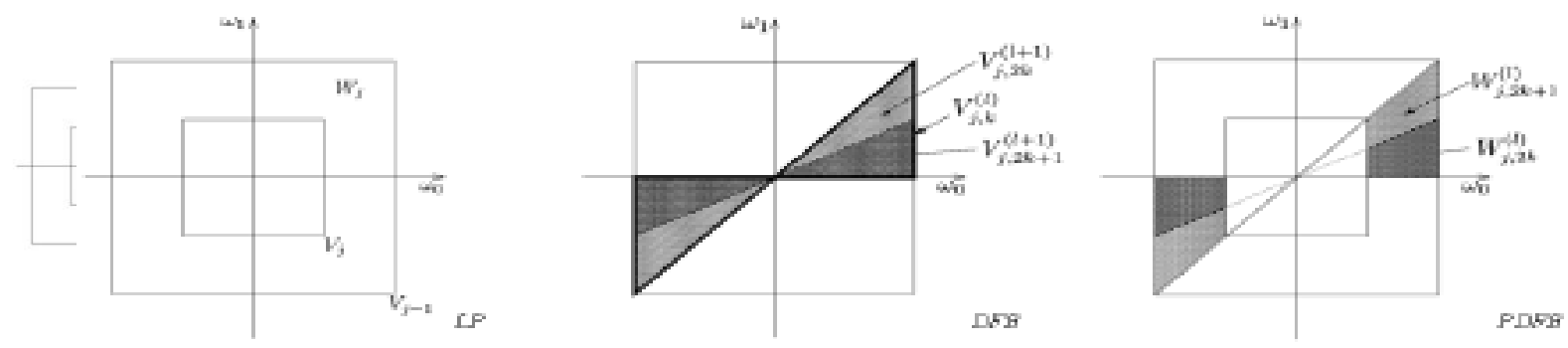

Fig.1 Generation of subspaces by the LP (left), DFB (middle) and PDFB (right);

$\mathrm{j}$ subspace index; $\mathrm{k}$ direction; 1 all directions

PDFB allows for different number of directions at each scale/resolution to nearly achieve critical sampling. As DFB is designed to capture high frequency components (representing directionality), the LP part of the PDFB permits sub band decomposition to avoid "leaking" of low frequencies into several directional sub bands, thus directional information can be captured efficiently. Fig. 1 illustrates the subspace splitting by respectively LP, DFB and PDFB. $V_{j}$ is a subspace, defined on a uniform grid with intervals $2^{\mathrm{j}} \times 2^{\mathrm{j}}$. The difference image in the LP carries the details necessary to increase the resolution from $\mathrm{V}_{\mathrm{j}}$ to $\mathrm{V}_{\mathrm{j}-1}$ on an image approximation; index $\mathrm{k}$ runs to all $2^{1}$ directions. As stated above, CTT offers the ability to choose the number of directions independently. We did early experiments with over 100 images, and it was proved, that the smoothness of the contours within an image is coupled with the spatial resolution of a desired scale. We found, that beyond a spatial resolution of $2^{8}$ pixels the application of the CTT carries no advantage compared with WVT in terms of information compaction. Thus, WVT has been used in this work instead the CTT for low resolution decomposition levels (scales). Therefore, the adopted image decomposition for compression purpose is then:

1. Four decomposition levels for image size $\leq 1024$ x 1024 pixels, where two scales are CTT, (with $\mathrm{l}=16$ directions) and the remaining two are WVT (l=3). (See Fig. 2).

2. Five decomposition levels for image size $\leq 2048 \times 2048$ pixels, thus, there are three CTT levels (with l=16 directions) and two WVT levels (l=3); 
3. six decomposition levels for image size $\leq 4096$ × 4096 pixels, thus, there are four CTT levels (with $1=16$ directions) and two WVT levels (l=3).

In this work, images with size described from item 1. And have been chosen for this investigation and the test results are reported in the next section.

\section{EXPERIMENTAL RESULTS}

Compression results using WVT and CTT on 3 selected images ("Man", "Berry" and "Art") are reported in this Section. The evaluation between these approaches is performed using the criterion PSNR and Potential Information Loss (PIL).

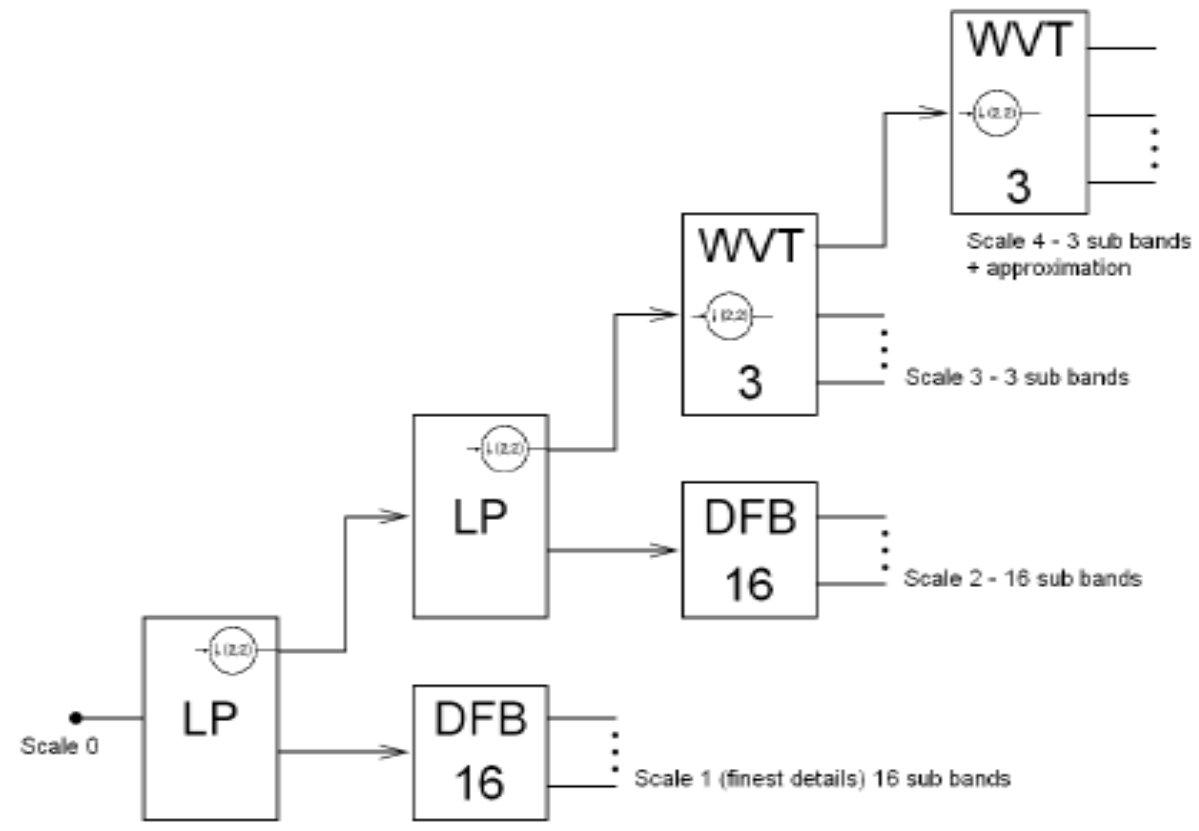

Fig. 2. CTT with four decomposition levels, WVT is used for the two coarse levels

PIL is a new metric, similar to the Kullback-Leibler distance, served for the evaluation of quality of the reconstructed image. It uses the probability density function of the image for evaluation. The histograms in Fig. 3 to Fig. 5 of the
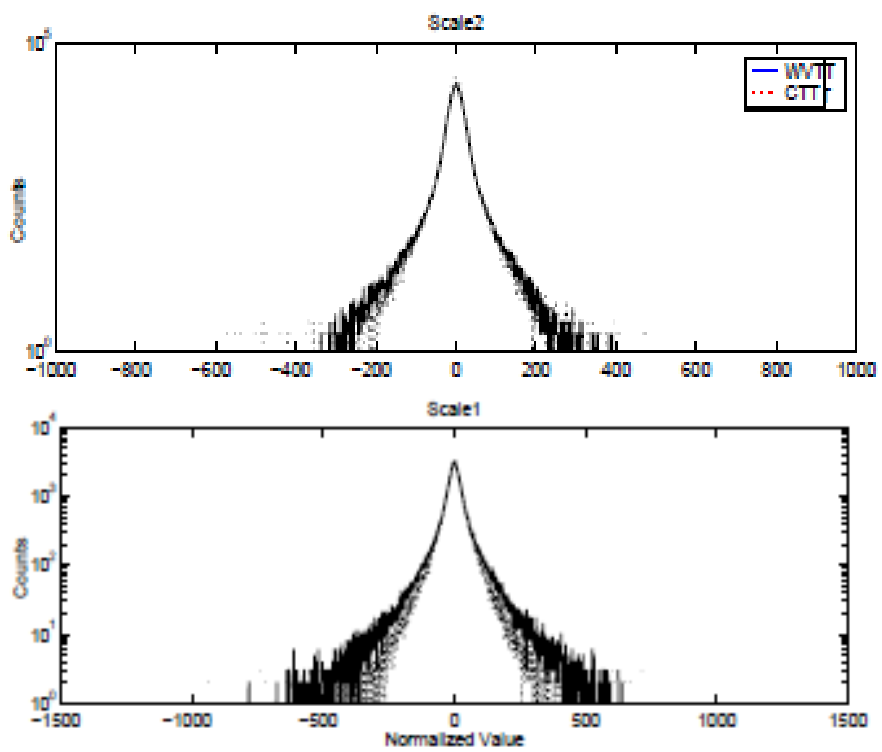

Fig. 3 The histograms of the coefficients "Man" image.

reconstructed and those of the original images are used for the calculation of PIL by means of the difference between both histograms (i.e. by computing the sum of the relative differences between all gray level counts). The original 
Vol. 8, Issue 4, April 2019

images are decomposed using CTT (see item 1. and 2. in Section 2) and WVT. Then, simple hard thresholding is performed over delcared insignificant coefficients (with amplitude $<$ a threshold). The images are reconstructed from the remaining significant coefficients and the reconstruction errors are derived. An arithmetic coder [20] is used then to encode the remaining coefficients and the factor between the coefficient sizes from the original and the encoded images are used to derive the compression ratios. Experimental results are presented in Table 1. The number of maintained significant coefficients using CTT is up to $20 \%$ smaller; the image quality gets better up to $1 \mathrm{~dB}$ (according to PSNR) than for WVT. Thus, the resulting compression ratio is slightly increased using CTT for an acceptable visual image quality. Fig. 6 depicts the original selected high resolution images "Man", "Berry" and "Art". Fig. 7, 8 and 9 present the resulting reconstructed images. All (a) labeled sub-figures are the WVT results. The WVT artifacts (image quality) can be seen in zoomed regions at the (b) labeled sub-figures. The (c) labeled sub-figures concern to the CTT results. The CTT image quality can be verified in zoomed regions (d). Additionally to the improved information compaction by CTT, visual quality preservation (better representation of edges) can be seen from the zoomed images (d) compared to WVT results (b). A reason for that can be noticed from Fig. 4, such that CTT
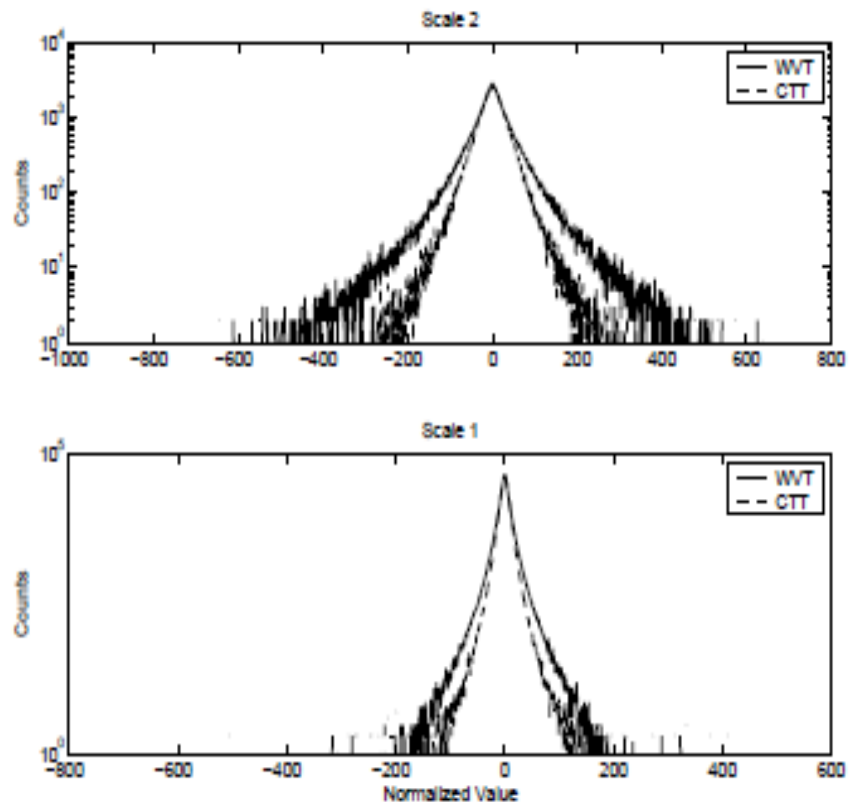

Fig.4 The histograms of the coefficients "Berry" image
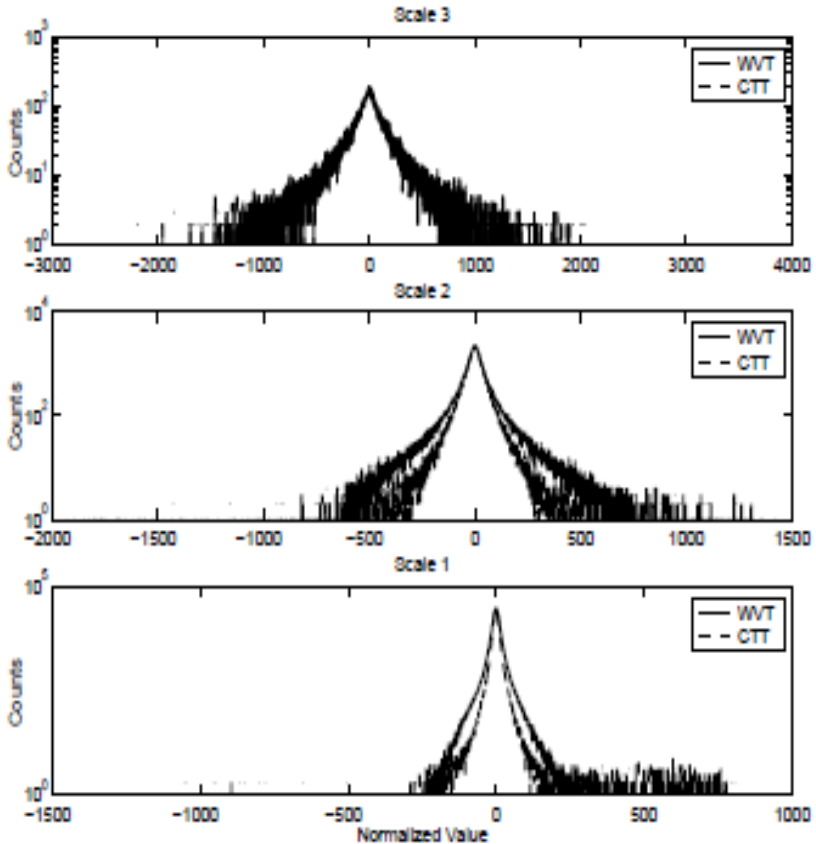

Fig. 5 The histograms of the coefficients "Art" image 
Vol. 8, Issue 4, April 2019

The original images are decomposed using CTT (see item in Section 2) and WVT. Then, simple hard thresh-olding is performed over declare insignificant coefficients (with amplitude < a threshold). The images are reconstructed from the remaining significant coefficients and the reconstruction errors are derived. An arithmetic coder [20] is used then to encode the remaining coefficients and the factor between the coefficient sizes from the original and the encoded images are used to derive the compression ratios. Experimental results are presented in Table 1. The number of maintained significant coefficients using CTT is up to $20 \%$ smaller; the image quality gets better up to $1 \mathrm{~dB}$ (according to PSNR) than for WVT. Thus, the resulting compression ratio is slightly in-creased using CTT for an acceptable visual image quality.

Table 1: Number of maintained coefficients, PSNR, PIL and CR for the test images

\begin{tabular}{|c|l|c|c|c|c|c|}
\hline $\begin{array}{c}\text { Sr. } \\
\text { NO }\end{array}$ & \multicolumn{1}{|c|}{ Images } & $\begin{array}{c}\text { Image } \\
\text { Size }\end{array}$ & $\begin{array}{c}\text { Nb, of Maintained } \\
\text { Coefficients }\end{array}$ & $\begin{array}{c}\text { PSNR } \\
\text { dB }\end{array}$ & PIL & $\begin{array}{c}\text { Compression } \\
\text { Ratio }\end{array}$ \\
\hline 1 & "Man" by WVT & $1 \mathrm{k}$ x 1k & 48522 & 24.5 & 0.62 & 2.8 \\
\hline 2 & "Man" by CTT & $1 \mathrm{k}$ x 1k & 43242 & 25.5 & 0.42 & 3.2 \\
\hline 3 & "Berry" by WVT & $1 \mathrm{k}$ x 1k & 5869 & 25.9 & 0.24 & 28.1 \\
\hline 4 & "Berry" by CTT & $1 \mathrm{k} \mathrm{x} \mathrm{1k}$ & 4668 & 26.4 & 0.20 & 29.3 \\
\hline 5 & "Art" by WVT & $2 \mathrm{k}$ x 2k & 16855 & 23.0 & 0.3 & 31.4 \\
\hline 6 & "Art" by CTT & $2 \mathrm{k}$ x 2k & 15248 & 24.1 & 0.3 & 33.1 \\
\hline
\end{tabular}

Fig. 6 depicts the original selected high resolution images "Man", "Berry" and "Art". Fig. 7, 8 and 9 present the resulting reconstructed images. All (a) labeled sub-figures are the WVT results. The WVT artifacts (image quality) can be seen in zoomed regions at the (b) labeled sub-figures. The (c) labeled sub-figures concern to the CTT results. The CTT image quality can be verified in zoomed regions (d).
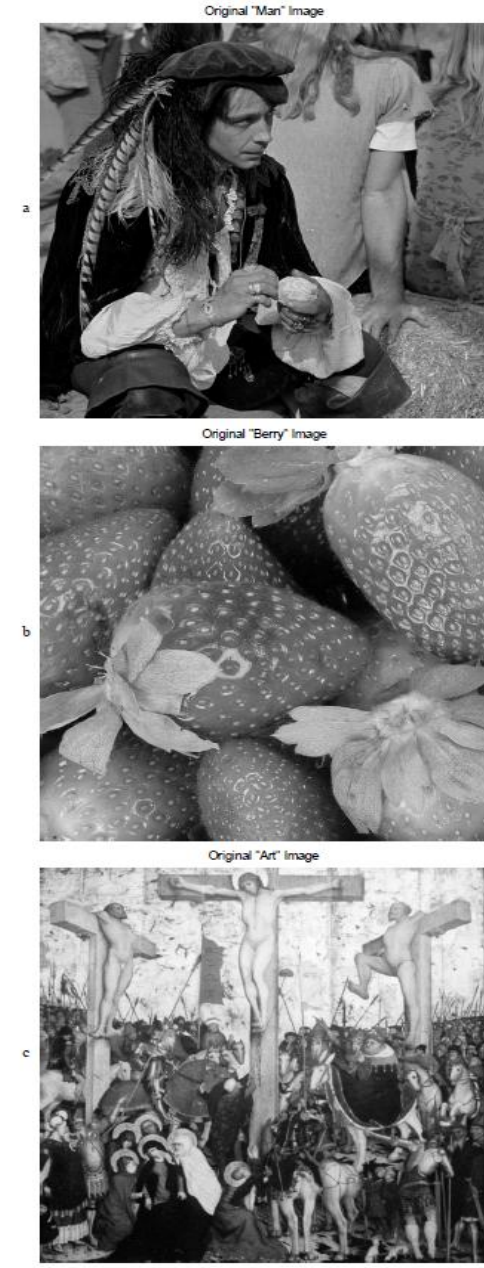

Fig. 6. The original images.

Fig. 7. Reconstructed "Man" images (at right).
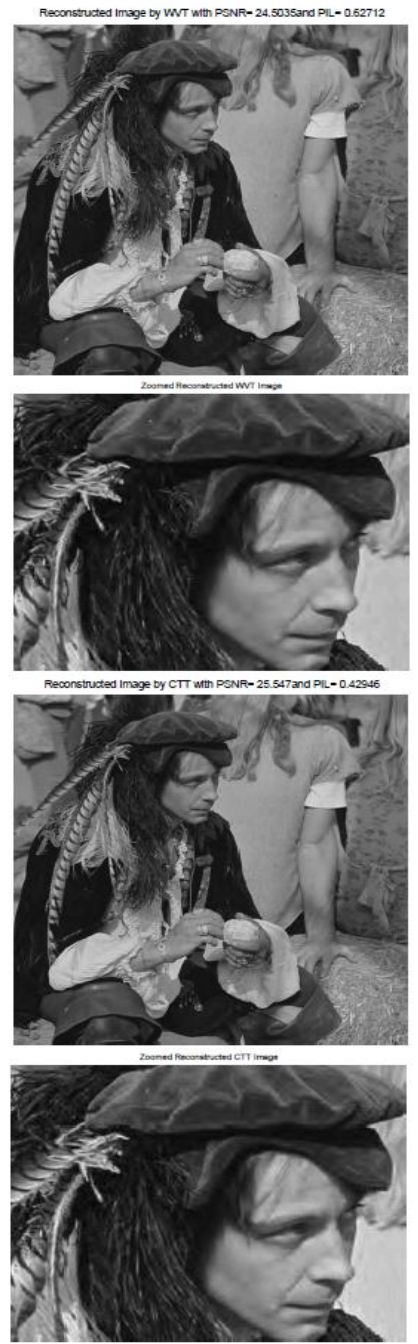
Additionally to the improved information compaction by CTT, visual quality preservation (better representation of edges) can be seen from the zoomed images (d) compared to WVT results (b). A reason for that can be noticed from Fig. 4, such that CTT provides a sparser representation of the images at fine scales and therefore, compacts them in fewer coefficients. At coarse scales, usually for $\leq 256$ × 256 pixel-images, WVT can out-perform CTT as object geometry is simplified and therefore, contours are not smooth anymore.

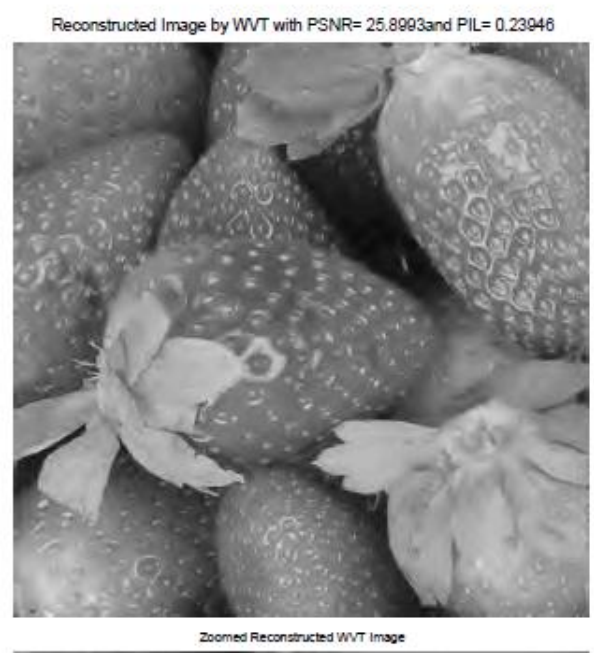

Fig.8 Resulting reconstructed images

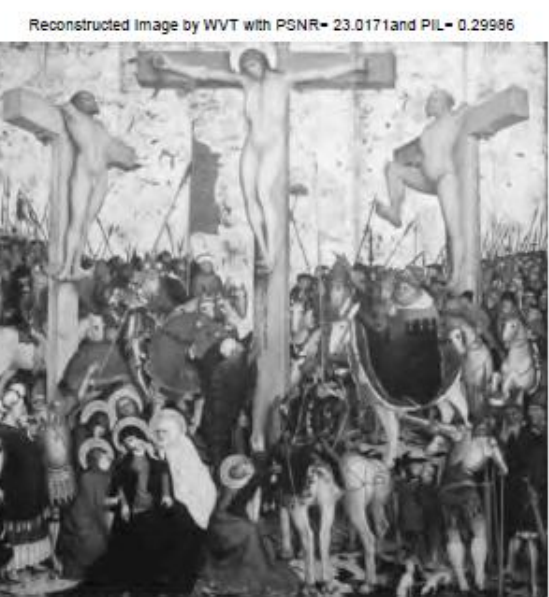

zoomed fesconetruces wit mose

Fig.9 Resulting reconstructed images

\section{CONCLUSION AND PERSPECTIVES}

This paper investigates the potential of the ConTourlet Transform (CTT) for compression of high resolution images ( $\geq$ 1 million pixels). The experimental results show a promising perspective as CTT provides a more compact representation of the information compared to that of the WaVelet Transform (WVT) used in the compression standard JPEG2000. This is figured out by the histograms of the coefficients at Fig. 3 to Fig. 5, which mostly shows the CTT inside the WVT curves. Furthermore, CTT shows less information loss and artifacts on the reconstructed images after a simple hard thresholding of the transformed coefficients. Indeed, WVT exhibits a large number of coefficients for representing smooth contours, which are usually present in high resolution images. However, WVT compacts better the information for the coarse resolution (up to $2^{8}$ pixel-image) as the geometry, usually, gets simplified. Therefore, a combination CTT-WVT (CTT for finest resolution and WVT for coarse resolution) seems to be good candidate for a new compression codec. Also a metric should be developed to find the objective best scale for the turnover from CTT to WVT processing. Investigations are still needed to recognize the adequate entropy coder for the resulting coefficients. Indeed, the hierarchical structure exploited by the JPEG $2000 \mathrm{codec}$ is not explicitly valid for our combination of methods. In a future work the combination of the coefficients from both transforms into zero trees (representing the inter-scale dependencies) will be studied.

\section{REFERENCES}

[1]. A.N. Belbachir and H. Bischof. On-Board Data Compression: Noise and Complexity Relate Aspects. Technical Report Number 75, PRIP, TU Vienna, 2

[2]. A.N. Belbachir, H. Bischof and F. Kerschbaum. A Data Compression Concept for Space Applications DSP-SPE'00, IEEE Digital Signal Proc. Workshop in Hunt, TX, USA, Oct. 2000.

[3]. P. J. Burt, E. H. Adelson. The Laplacian pyramid as a compact image coder. IEEE Trans. Commun, Vol.31(4) : 532-540, April 1983.

[4]. E.J. Candes and D.L. Donoho. Curvelets - A Surprisingliy Ef-fective Non-Adaptive Representation for Objects with Edges. In Curve and Surface Fitting, A. Cohen, C. Rabut, and L.L. Schu-maker, Eds. Saint Malo: Vanderbilt University, 1999.

[5]. E. J. Candes and D. L. Donoho. Ridgelets: a Key to Higher-Dimensional Intermittency? Phil. Trans. R. Soc. Lond. A., pp. 2495-2509, 1999.

[6]. M.N. Do and M. Vetterli. Orthonormal Finite Ridgelet Trans-form for Image Compression. ICIP'2000, Vancouver,Canada, September 2000.

[7]. D.Y.D Po \& M. Do. Directional multiscale Modeling of Images using the contourlet transform. IEE trans on Image processing, Draft version of june

[8]. J.L Starck D.L Donoho and E. J Candes. Astronomical Image Representation by the curvelet Transform. Astronomy and Astrophysics 398, 785-800:2003 